\title{
A Theoretical Study on the Effects of Interference on UWB Multiple Access Impulse Radio *
}

\author{
Ali Taha, Keith M. Chugg \\ Department of Electrical Engineering-Systems \\ University of Southern California \\ Los Angeles, CA 90089-2565 \\ taha@usc.edu , chugg@usc.edu
}

\begin{abstract}
Ultra wide bandwidth impulse radio occupies huge bandwidth from near DC to up to a few GHz. This suggests that many coexisting communication systems working simultaneously at different regions of impulse radio's bandwidth cause interference. In this paper, the effects of an arbitrary external interference as SNR and bit error rate degradation of multiple access $U W B$ radio versus interference frequency is theoretically evaluated and pulse shape design for narrow band interference rejection is presented. Using doublet pulses [1], it is shown how narrow bandwidth interference is mitigated significantly. Effects of amplitude mismatches and gap time offset from the nominal value in a doublet pulse are investigated.
\end{abstract}

\section{1: Introduction}

Digital impulse radio enjoys an excellent multipath resolution capability up to a fraction of a nanosecond, due to its huge bandwidth. On the other hand, occupying a vast amount of bandwidth causes new challenges for this kind of radio, since there are many other communication systems working at different portions of UWB radio bandwidth simultaneously [2]. This introduces external interference sources which cause performance degradation. In this paper, we investigate SNR and bit error rate degradation in terms of the interference frequency and show how using different pulse shapes can mitigate some narrow bandwidth interferers. Moreover, we derive the relationship between gap time in doublet pulses and narrow bandwidth interference rejection, so that with careful design of such pulses we are able to mitigate a strong disturbing narrow band interference simultaneously present in the communication environment.

\footnotetext{
*This work has been supported in part by Army Research Office and National Science Foundation under the grants DAAD1901-1-0477 and ANI-9730556, respectively.
}

In Section II, the problem is mathematically modelled and SNR and bit error rate in the presence of an arbitrary random wide-sense stationary external interference is computed. Pulse shape design in order to mitigate narrow bandwidth interference is discussed in Section III. Section IV discusses the effects of amplitude mismatches and gap time offset from the nominal value on the location and magnitude of the generated nulls in the energy spectral density of the pulse shape used, followed by conclusion remarks in Section V.

\section{2: Mathematical Formulation}

Sending one pulse in each frame period $T_{f}$ [3], [4], the transmitted signal of the $k$ th user with antipodal data modulation is

$$
S_{\text {trans }}^{k}(t)=\sum_{j} D_{j}^{k} w_{\text {trans }}\left(t-j T_{f}-c_{j}^{k} T_{c}\right)
$$

where $w_{\text {trans }}(t)$ is the transmitted waveform in the channel and $c_{j}^{k}$ is the time-hopping code of the $k$ th user in the $j$ th frame with $T_{c}$ representing a chip time. Also, $D_{j}^{k}$ accounts for the $k$ th user's symbol in the $j$ th frame and $D_{j}^{k} \in\{-1,1\}$. In order to obtain processing gain, we send $N_{s}$ pulses per data symbol; therefore, $D_{i N_{s}}^{k}=D_{i N_{s}+1}^{k}=\cdots=D_{(i+1) N_{s}-1}^{k}=d_{i}^{1}$.

We model the received signal at the output of the receiver's antenna subsystem as

$$
r(t)=\sum_{k=1}^{N_{u}} g_{k} S_{r e c}^{k}\left(t-\tau_{k}\right)+I(t)+n(t)
$$

where $S_{r e c}^{k}(t)=\sum_{j} D_{j}^{k} w\left(t-j T_{f}-c_{j}^{k} T_{c}\right)$ is the $k$ th user received signal with $w(t)$ representing the received waveform at the output of the receiver's antenna subsystem. The amplitude of the $k$ th user is denoted as $g_{k}$. Fig. 1 shows the block diagram of the receiver. The received pulse shape scaled by the amplitude of 
the user of interest is used as template waveform [5] at the receiver's correlator. As can be seen, the receiver

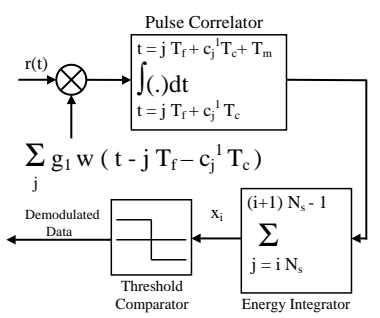

Figure 1: Receiver Block Diagram

computes the decision statistic $x_{i}$ for the $i$ th data symbol as $x_{i}=\int_{t=i N_{s} T_{f}}^{t=(i+1) N_{s} T_{f}} r(t) \sum_{j=i N_{s}}^{(i+1) N_{s}-1} g_{1} w\left(t-j T_{f}-\right.$ $\left.c_{j}^{1} T_{c}\right) d t$. If $x_{i}>0$, then the decision will be in the favor of hypothesis $H_{1}$, i.e., the demodulated data is declared as a 1 , otherwise hypothesis $H_{-1}$ is stated to be true. Without loss of generality, we assume that the delay of the user of interest $\tau_{1}=0$, so each other user's delay with respect to the delay of the user of interest can be written as $\tau_{k}=\alpha_{k} T_{f}+\beta_{k}$, where $\beta_{k}$ is in $\left[\frac{-T_{f}}{2}, \frac{T_{f}}{2}\right]$. After some manipulations, we get

$$
x_{i}=s_{i}+c_{i}+e_{i}+n_{i}
$$

where

$$
s_{i}=N_{s} g_{1}^{2} d_{i}^{1} R_{w}(0)
$$

corresponds to the signal of the user of interest, and $R_{w}(\tau)=\int_{-\infty}^{\infty} w(t+\tau) w(t) d t$. The second term on the right hand side of (3),

$$
c_{i}=\sum_{j=i N_{s}}^{(i+1) N_{s}-1} \sum_{k=2}^{N_{u}} g_{1} g_{k} D_{j-\alpha_{k}}^{k} R_{w}\left(\left(c_{j}^{1}-c_{j-\alpha_{k}}^{k}\right) T_{c}-\beta_{k}\right)
$$

accounts for the cross-talk due to the multiple-access interference. Also,

$$
\begin{aligned}
e_{i}=\sum_{j=i N_{s}}^{(i+1) N_{s}-1} \int_{i N_{s} T_{f}-j T_{f}-c_{j}^{1} T_{c}}^{(i+1) N_{s} T_{f}-j T_{f}-c_{j}^{1} T_{c}} \\
g_{1} I\left(t+j T_{f}+c_{j}^{1} T_{c}\right) w(t) d t
\end{aligned}
$$

represents the effect of the external interference. The last term on the right hand side of (3),

$$
\begin{aligned}
n_{i}=\sum_{j=i N_{s}}^{(i+1) N_{s}-1} \int_{i N_{s} T_{f}-j T_{f}-c_{j}^{1} T_{c}}^{(i+1) N_{s} T_{f}-j T_{f}-c_{j}^{1} T_{c}} \\
g_{1} n\left(t+j T_{f}+c_{j}^{1} T_{c}\right) w(t) d t
\end{aligned}
$$

is due to the random Gaussian noise with variance $\sigma_{n_{i}}^{2}=N_{s} g_{1}^{2} \frac{N_{0}}{2} R_{w}(0)$ at the input of the threshold detector.

Since the support of $w(t)$ is $\left[0, T_{m}\right]$, and the interval integral $\left[i N_{s} T_{f}-j T_{f}-c_{j}^{1} T_{c},(i+1) N_{s} T_{f}-j T_{f}-c_{j}^{1} T_{c}\right]$, contains this support for any integer $j$ in $\left[i N_{s},(i+\right.$ 1) $N_{s}-1$ ], we can extend the integral interval in (6) to the whole real line, hence, the variance of $e_{i}$ can be computed as

$$
\begin{array}{r}
\lambda_{i}=E\left\{e_{i}^{2}\right\}=g_{1}^{2} \sum_{j=i N_{s}}^{(i+1) N_{s}-1} \sum_{j^{\prime}=i N_{s}}^{(i+1) N_{s}-1} \int_{-\infty}^{\infty} R_{I}(\tau) \\
R_{w}\left(\tau-\left(j-j^{\prime}\right) T_{f}-\left(c_{j}^{1}-c_{j^{\prime}}^{1}\right) T_{c}\right) d \tau
\end{array}
$$

where $R_{I}(\tau)=E(I(t+\tau) I(t))$. Under certain circumstances when the time-hopping range is restricted to the interval $\left[0, \frac{T_{f}}{2}-T_{m}\right]$, and $R_{I}(\tau)=0$ for $|\tau|>\frac{T_{f}}{2}$, $E\left\{e_{i}^{2}\right\}$ can be simplified to

$$
E\left\{e_{i}^{2}\right\}=g_{1}^{2} N_{s} \int_{-\infty}^{\infty} R_{I}(\tau) R_{w}(\tau) d \tau
$$

The above formula is also valid without any restriction on the time-hopping range or any assumption on the autocorrelation function of $I(t)$, as long as $N_{s}=1$.

Using Parseval's Theorem, (8) can be written as

$$
\lambda_{i}=g_{1}^{2} \sum_{j=i N_{s}}^{(i+1) N_{s}-1} \sum_{\substack{j^{\prime}=i N_{s} \\ j 2 \pi f\left(\left(j-j^{\prime}\right) T_{f}+\left(c_{j}^{1}-c_{j^{\prime}}^{1}\right) T_{c}\right)} d f}^{\infty(i+1) N_{s}-1} \int_{-\infty}^{\infty} S_{I}(f) S_{w}(f)
$$

This means that by careful design of the pulse shape $w(t)$, we can mitigate interference by generating nulls in the spectral density of the pulse shape around frequencies where the interference is strong.

Being perfectly synchronized to the time of arrival and time-hopping code of the user of interest at the receiver's correlator, the SNR at the output of the user of interest's receiver is computed as

$$
\operatorname{SNR}\left(N_{u}\right)=\int_{0}^{\infty} \frac{N_{s}^{2} g_{1}^{4} R_{w}^{2}(0)}{P_{M A I}+\lambda+\sigma_{n_{i}}^{2}} f(\lambda) d \lambda
$$

where $f(\lambda)$ is the probability density function of the external interference variance given by (8), because this variance changes depending on the starting position of $c_{j}^{1}$. Here, $P_{M A I}=E\left\{c_{i}^{2}\right\}=N_{s} \sum_{k=2}^{N_{u}}\left(g_{1} g_{k}\right)^{2} \sigma^{2}$ represents the multiple access interference variance using the central limit theorem when the time-hopping range is restricted to $\left[0, \frac{T_{f}}{2}-T_{m}\right]$ and $\sigma^{2}=\frac{1}{T_{f}} \int_{-\infty}^{\infty} R_{w}^{2}(x) d x$.

Consider the case where $R_{I}(\tau)=\frac{I_{0}{ }^{2}}{2} \cos \left(2 \pi f_{0} \tau\right)$, which corresponds to the tone interference $I(t)=$ 
$I_{0} \cos \left(2 \pi f_{0} t\right)$. Fig. 2 shows the effect of interference on $\operatorname{SNR}(20)$ as SNR degradation versus the signal to interference ratio (SIR) at the receiver's front end when the received pulse shape is the second order derivative of Gaussian shown in Fig. 3. Twenty active users are communicating at $1 \mathrm{Mbps}$ data rate. The operating $\operatorname{SNR}(20)$ has been assumed $10 \mathrm{~dB}$ in the absence of interference.

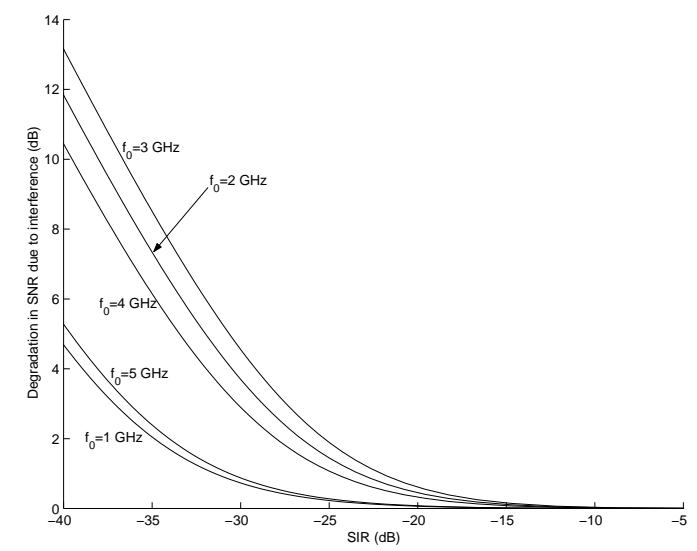

Figure 2: Effect of tone interference on the SNR

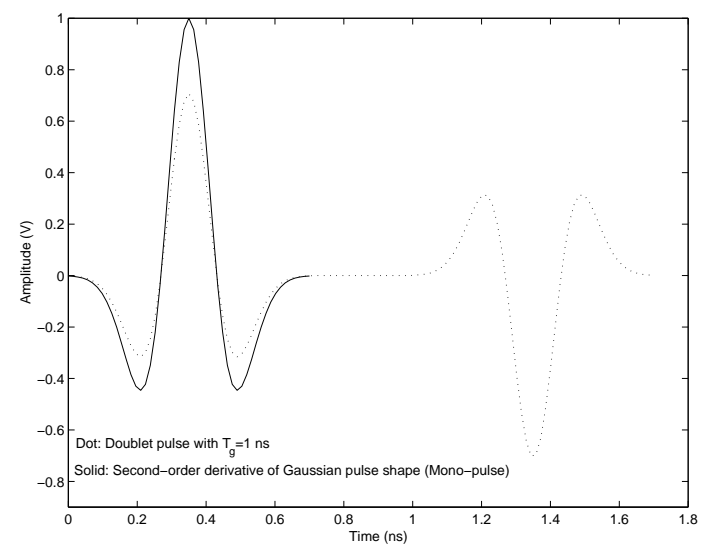

Figure 3: Second order derivative of Gaussian and doublet pulses

Due to the symmetry of the problem the bit error probability can be computed as $\operatorname{Pr} .\{\epsilon\}=\operatorname{Pr} .\left\{\epsilon \mid d_{i}^{1}=\right.$ $1\}=\operatorname{Pr} .\left\{x_{i}<0 \mid d_{i}^{1}=1\right\}$. Using (3) and (4) and replacing $d_{i}^{1}=1, \operatorname{Pr} .\{\epsilon\}=\operatorname{Pr} .\left\{c_{i}+e_{i}+n_{i}<-N_{s} g_{1}^{2} R_{w}(0)\right\}$. Applying the central limit theorem, and assuming perfect power control, i.e., $\frac{g_{k}^{2}}{g_{1}^{2}}=1$, we get

$\operatorname{Pr} .\{\epsilon\}=\int_{0}^{\infty} Q\left(\frac{R_{w}(0)}{\sqrt{\frac{\left(N_{u}-1\right) \sigma^{2}}{N_{s}}+\frac{\lambda}{g_{1}^{4} N_{s}^{2}}+\frac{R_{w}(0)}{N_{s} g_{1}^{2}} \frac{N_{0}}{2}}}\right)$
Fig. 4 shows the bit error rate performance of multipleaccess digital impulse radio in the presence of an external tone interference with SIR around $-37 \mathrm{~dB}$ versus the frequency of the interference when 20 users are communicating at $1 \mathrm{Mbps}$ data rate. The bit error rate in the absence of interference is equal to $7.8270 \mathrm{e}-4$, which corresponds to $\operatorname{SNR}(20)=10 \mathrm{~dB}$. The solid curve in Fig. 4

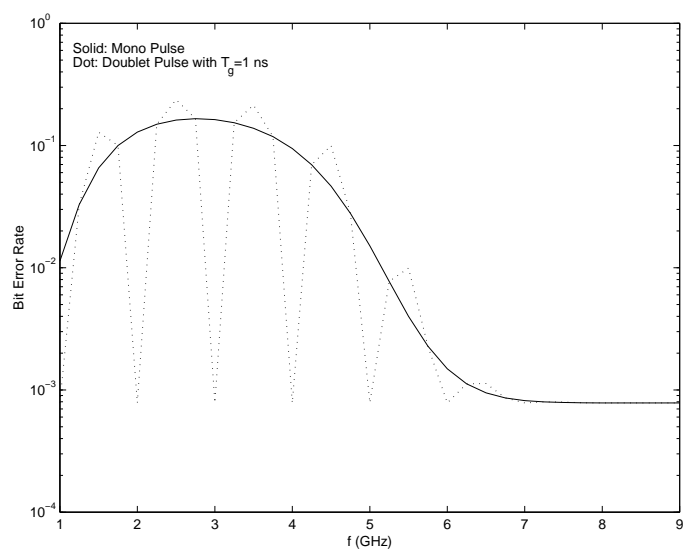

Figure 4: Bit error rate versus the interference frequency

corresponds to using a single second-order derivative of Gaussian pulse shape. As can be seen, the bit error rate performance in the presence of interference degrades more severely at frequencies where the spectral density of the second-order derivative of Gaussian pulse shape shown in Fig. 5 is stronger. The dotted curve in Fig. 4 corresponds to using a doublet pulse shape, which will be described in Section III.

Since $E\left\{D_{j}^{k}\right\}=0$, the discrete part of the power spectral density consisting of spectral lines at integer multiples of $\frac{1}{T_{f}}$ vanishes [6], and the spectral density of the modulated time-hopped signal, $s(t)=$ $\sum_{k=1}^{N_{u}} g_{k} \sum_{j} D_{j}^{k} w\left(t-j T_{f}-c_{j}^{k} T_{c}-\tau_{k}\right)$, can be computed as $S_{s}(f)=\frac{|W(f)|^{2}}{T_{f}} \sum_{k=1}^{N_{u}} g_{k}^{2}$. Therefore, the shape of the spectral density of the received signal is just a scaled version of the energy spectral density of the received pulse shape $w(t)$.

As can be observed from Fig.'s 2 and 4, the tone interference degrades the performance differently depending on which portion of UWB radio operating bandwidth it hits. Fig. 5 demonstrates the normalized energy spectrum of the second-order derivative of Gaussian received pulse shape shown in Fig. 3. At around $f_{0}=3 \mathrm{GHz}$, where the frequency content of the received pulse spectrum is very strong, the tone interference degrades the performance much more severely. Also at places where the frequency contents have al- 
most the same strengths, e.g. at $1 \mathrm{GHz}$ and $5 \mathrm{GHz}$, the degradations arising from the tone interferers are almost the same.

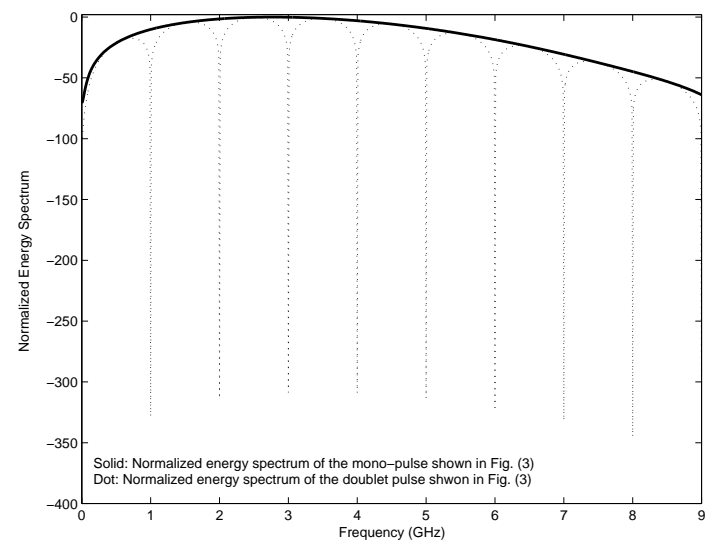

Figure 5: Normalized energy spectrum of mono and doublet waveforms shown in Fig. 3

\section{3: Interference Rejection}

A doublet pulse consists of two received waveforms separated from each other by $T_{g}$ time shift and with opposite amplitudes, i.e., $w_{d}(t)=\frac{1}{\sqrt{2}}\left(w(t)-w\left(t-T_{g}\right)\right)$. Fig. 3 shows such a pulse. The spectral amplitude of such a pulse shape can be computed as

$$
\left|W_{d}(f)\right|^{2}=2|W(f)|^{2} \sin ^{2}\left(\pi f T_{g}\right)
$$

where $|W(f)|^{2}$ is the magnitude squared of a single received pulse spectrum. Therefore, the spectrum has nulls at $f=\frac{k}{T_{g}}$ for any integer $k$ as illustrated in Fig. 5 . By controlling $T_{g}$, we can generate nulls at specific frequencies to mitigate interferers around those frequencies. We can adjust the nulls by changing $T_{g}$ in order to reject powerful narrow band interferers existing simultaneously at operating bandwidth of UWB radio in a particular environment. For example, if we set $T_{g}=1$ ns, we can completely remove all the interferers whose frequencies are multiple integers of $1 \mathrm{GHz}$. This will lead to neither any SNR nor bit error rate performance degradation in Fig.'s 2 and 4 for $f_{0}=1,2,3, \cdots, 9$ $\mathrm{GHz}$, regardless of how strong a tone interference at such frequencies is.

Fig. 6 shows the SNR degradation in the presence of an external interference with flat power spectral density over $B=50 \mathrm{MHz}$ and $B=100 \mathrm{MHz}$ bandwidth and $2 \mathrm{GHz}$ center frequency when $20 \mathrm{UWB}$ users are simultaneously communicating at $1 \mathrm{Mbps}$ data rate. The operating $\operatorname{SNR}(20)$ has been assumed $10 \mathrm{~dB}$ in the absence of external interference. As can be seen, there is

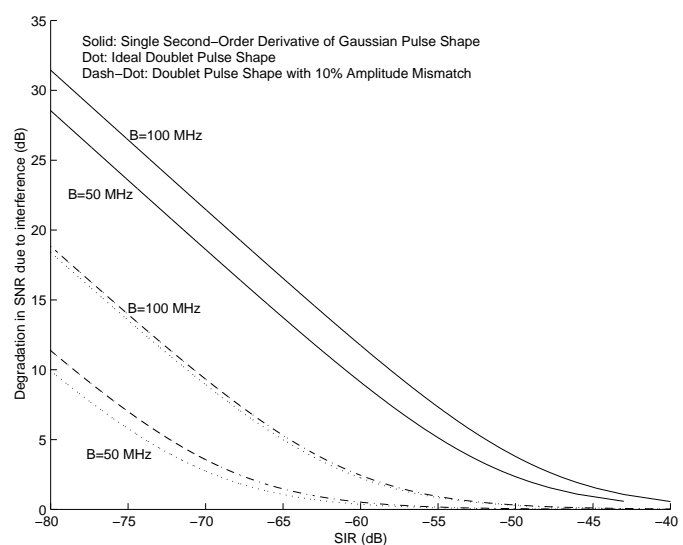

Figure 6: SNR degradation due to a narrow band interference with or without using a doublet pulse

a gain of $13 \mathrm{~dB}$ and $18.6 \mathrm{~dB}$ using a properly designed doublet pulse compared to just using a single pulse shape when the interference is $80 \mathrm{~dB}$ stronger than the signal of the user of interest with $B=100 \mathrm{MHz}$ and $B=50 \mathrm{MHz}$ bandwidth, repectively. It is worth noting that assuming a flat power spectral density for a narrow band interference over a fixed bandwidth at a specific center frequency, actually represents a harsh situation, because the interference power is equally spread over its bandwidth, which hurts our system more severely if the generated notch by a doublet pulse is not wide enough in the frequency domain. On the contrary, a narrow band interference over a fixed bandwidth with power mostly concentrated around its center frequency, can be easily mitigated by adjusting the notch sharply in the middle of its bandwidth.

It can be shown that the asymptotic performance gain in Fig. 6, obtained by using a proper doublet pulse, $w_{d}(t)$, over a mono-pulse, $w(t)$, when the interference becomes very strong is

$$
G=10 \log \left(\frac{\int_{B 1}^{B 2} S_{w}(f) d f}{\int_{B 1}^{B 2} S_{w_{d}}(f) d f}\right)
$$

where $\frac{B 1+B 2}{2}$ is the narrow band interference center frequency with bandwidth $B=B 2-B 1$. This asymptotic gain is $13.1 \mathrm{~dB}$ and $19.1 \mathrm{~dB}$ for the cases of $B=100$ $\mathrm{MHz}$ and $B=50 \mathrm{MHz}$ shown in Fig. 6, respectively.

To mitigate two different external interferers at two different frequencies, we can design $T_{g}=\frac{1}{G . C . D .\left\{f_{1}, f_{2}\right\}}$, where G.C.D. represents the greatest common devisor operation over those external interferers' center frequencies. 


\section{4: Effect of Mismatches}

In this section, the effect of mismatches in the positive and negative amplitudes of a doublet pulse along with having an offset in the gap time from its nominal value are investigated. To model these effects, doublet pulse is expressed in the form: $w_{d}(t)=$ $w(t)-\alpha w\left(t-\beta T_{g}\right)$ where $w(t)$ is the received pulse shape and $\alpha$ and $\beta$ are used to model amplitude mismatch and gap time offset in the doublet pulse, respectively. Therefore, the spectral amplitude squared of the doublet pulse is $\left|W_{d}(f)\right|^{2}=|W(f)|^{2} C(f)$ where $C(f)=\left[1-2 \alpha \cos \left(2 \pi \beta T_{g} f\right)+\alpha^{2}\right]$. In order to generate perfect nulls at some frequency $f_{0}$, we need to have $C\left(f_{0}\right)=0$ for an arbitrary pulse shape $w(t)$. Hence, $C\left(f_{0}\right)=0 \Rightarrow \cos \left(2 \pi \beta T_{g} f_{0}\right)=\frac{1+\alpha^{2}}{2 \alpha}$. Since $\alpha$ is a number around 1 to represent the amplitude mismatch between the positive and negative polarities in the doublet pulse; therefore, $\alpha>0$, and $\frac{1+\alpha^{2}}{2 \alpha} \geq 1$. This means that perfect nulls can be generated only when there is no amplitude mismatch or when $\alpha=1$; however, by finding the minima of $C(f)$, we can locate the position of deep nulls in the frequency spectrum: $\frac{d C(f)}{d f}=0 \Rightarrow f=\frac{k}{2 \beta T_{g}}$, where $k$ is an integer.

When $k$ is an even integer, we get $\left|W_{d}\left(\frac{k}{2 \beta T_{g}}\right)\right|^{2}=$ $\left|W\left(\frac{k}{2 \beta T_{g}}\right)\right|^{2}(1-\alpha)^{2}$, and when $k$ is an odd integer, $C\left(\frac{k}{2 \beta T_{g}}\right)=(1+\alpha)^{2}$, which corresponds to the local maxima of $C(f)$. Hence, nulls can be generated only at even multiples of $\frac{1}{2 \beta T_{g}}$, which is equivalent to any integer multiple of $\frac{1}{\beta T_{g}}$. Fig. 6 shows that little performance degradation is observed when using a non-ideal doublet pulse.

\section{5: Conclusion}

Using a huge bandwidth, digital impulse radio has to face interference from external sources working at different portions of its bandwidth. Interference coming from these external sources degrades the UWB radio perfromance. Depending on the frequency of narrow band interferers, they degrade the performance differently. Those with frequencies concentrated at regions where the UWB radio pulse has stronger frequency contents, degrade the performance more severely. By careful design of UWB pulse shape, we can mitigate the narrow bandwidth interference. This can be achieved with careful design of gap time between the two single pulses in a doublet pulse in order to generate nulls in the frequency domain where the interference is present; therefore, with this technique, we do not need to use any additional expensive filter to reject narrow band- width interference.

Studies of amplitude mismatches and gap time offset show how the location and magnitude of the nulls are affected by these factors when a doublet pulse is used in order to avoid narrow bandwidth interference. The results obtained in this paper are general and not limited to the pulse shapes shown in Fig. 3, and can be applied to any arbitrary pulse shape as may be required to meet spectral masked imposed by regulatory authorities.

\section{Acknowledgement}

The authors wish to thank Professor Robert A. Scholtz from University of Southern California for his comments and reading the first draft of this paper.

\section{References}

[1] M. Hamalainen, V. Hovinen, J. Iinatti, M. Latvaaho, "In-Band Interference Power Caused by Different Kinds of UWB Signals at UMTS/WCDMA Frequency Bands," IEEE Radio and Wireless Conference, 2001.

[2] R. A. Scholtz, R. Weaver, E. Homier, J. Lee, P. Hilms, A. Taha, R. Wilson, "UWB Radio Deployment Challenges," IEEE Personal Indoor Mobile Radio Communications, London, U.K., September 2000 .

[3] Moe Z. Win, Robert A. Scholtz, "Ultra-Wide Bandwidth Time-Hopping Spread Spectrum Impulse Radio for Wireless Multiple-Access Communications," IEEE Transactions on Communications, Vol. 48, No. 4, April 2000.

[4] Ali Taha, Keith M. Chugg, "Multipath Diversity Reception of Wireless Multiple Access TimeHopping Digital Impulse Radio," IEEE Conference on Ultra Wideband Systems and Technologies, Baltimore, M.D., May 2002.

[5] Ali Taha, Keith M. Chugg, "On Designing the Optimal Template Waveform for UWB Impulse Radio in the Presence of Multipath," IEEE Conference on Ultra Wideband Systems and Technologies, Baltimore, M.D., May 2002.

[6] Moe Z. Win, "Spectral Density of Random TimeHopping Spread-Spectrum UWB Signals with Uniform Timing Jitter," IEEE Military Communications Conference Proceedings, Vol. 2, 1999. 